\title{
Comparison of DSP-based nonlinear equalizers for intra-channel nonlinearity compensation in coherent optical OFDM
}

\author{
Elias Giacoumidis, ${ }^{1}$ Sofien Mhatli, ${ }^{2}$ Tu Nguyen, ${ }^{3}$ Son T. Le, ${ }^{4}$ IVAn Aldaya, ${ }^{5}$ \\ Mary E. McCarthy, ${ }^{4}$ Andrew D. Ellis, ${ }^{4}$ Benjamin J. Eggleton, ${ }^{1}$ \\ ${ }^{1}$ Centre for Ultrahigh bandwidth Devices for Optical Systems (CUDOS) and Institute of Photonics and Optical Science (IPOS), School of Physics, \\ University of Sydney, NSW 2006, Sydney, Australia \\ 2 SERCOM-Lab, EPT Université de Carthage, La Marsa, Tunis 2078, Tunisia \\ ${ }^{3}$ FPM-UMONS, 31 Dolez, B-7000 Mons, Belgium \\ ${ }^{4}$ Aston Institute of Photonic Technologies (AIPT), Aston University, Birmingham, B4 7ET, England, UK \\ ${ }^{5}$ Physics Institute, State University of Campinas, 777, Campinas, Brazil \\ *Corresponding author: e.giacoumidis@physics.usyd.edu.au
}

Received 31 January 2016; revised XX Month, 2016; accepted XX Month XXXX; posted XX Month XXXX (Doc. ID XXXXX); published XX Month XXXX

\begin{abstract}
A novel versatile digital signal processing (DSP) based equalizer using support vector machine regression (SVR) is proposed for 16-quadrature amplitude modulated (16QAM) coherent optical orthogonal frequency-division multiplexing (CO-OFDM) and experimentally compared to traditional DSP-based deterministic fiber-induced nonlinearity equalizers (NLEs), namely the full-field digital back propagation (DBP) and the inverse Volterraseries transfer function based NLE (V-NLE). For $40-\mathrm{Gb} / \mathrm{s}$ 16-QAM CO-OFDM at $2000 \mathrm{~km}$, SVR-NLE extends the optimum launched optical power (LOP) by $4 \mathrm{~dB}$ compared to V-NLE by means of reduction of fiber nonlinearity. In comparison to full-field DBP at a LOP of $6 \mathrm{dBm}$ SVR-NLE outperforms by $\sim 1 \mathrm{~dB}$ in $\mathrm{Q}$-factor. In addition, SVR-NLE is the most computational efficient DSP-NLE. (C) 2015 Optical Society of America
\end{abstract}

OCIS codes: (060.2330) Fiber optic communications, (060.4080) Modulation, (060.1660) Coherent communications.

http://dx.doi.org/10.1364/OL.99.099999

Endeavors to surpass the Kerr-induced nonlinearity limit have been performed by either inserting an optical phase conjugator (OPC) at the middle point of the link [1] or using electronic-based nonlinearity compensators (NLC) such as digital back-propagation (DBP) placed in the receiver [2] or transmitter [3], phaseconjugated twin-waves (PC-TW) [4], and nonlinear equalizers (NLEs) based on the inverse Volterra-series transfer function (VNLE) [5]. Unfortunately, OPC significantly reduces the flexibility in an optically routed network requiring both symmetric $2^{\text {nd }}$ order chromatic dispersion (CD) and power evolution, DBP is extremely complex and PC-TW halves the transmission capacity. V-NLE has been considered as a simple and effective method for combating fiber nonlinearities, however it still requires a significant amount of floating-point multiplications. Additionally, in coherent communication systems the interaction between nonlinear phenomena, CD, and frequency fluctuations of source and local oscillators (LO) results in stochastic nonlinear distortion, which can be partially mitigated using either frequency referenced carriers [3] or nonlinear mapping based on statistical learning such as artificial neural networks (ANN) [6] and support vectors machines (SVM) [7].

On the other hand, coherent optical orthogonal frequencydivision multiplexing (CO-OFDM) is an excellent candidate for longhaul communications because of its high spectral efficiency and tolerance to $\mathrm{CD}$ and polarization-mode dispersion (PMD). However, due to its high peak-to-average power ratio (PAPR) the nonlinear cross-talk effects among subcarriers such as cross-phase modulation (XPM) and four-wave mixing (FWM) are enhanced, causing a stochastic-like interference to the extent of becoming an insurmountable obstacle. Owing to the vulnerability of CO-OFDM in nonlinear distortion, it is envisaged that NLC will enhance the capacity and transmission-reach in coherent optical core networks [8], thus avoiding highly dissipative regeneration electronics [3]. However, NLC feasibility demands the employment of versatile (i.e. independent from link parameters) techniques of low complexity for real-time applications.

In this letter, it is experimentally compared, for the first time, $\mathrm{V}$ NLE and full-field DBP-NLE with a novel SVM-based regression (SVR) NLE in 40-Gb/s 16-quadrature amplitude modulation (16QAM) CO-OFDM at $2000 \mathrm{~km}$. In contrast to nonlinear classifiers such as ANN [6] and SVM [7], SVR projects the obtained data on a hyperplane where constellation regions are easier to decode. It is shown that SVR-NLE can extend the optimum launched optical power (LOP) by $4 \mathrm{~dB}$ compared to both linear equalization and VNLE by means of reduction of fiber nonlinearity. In comparison to full-field DBP-NLE at a LOP of $6 \mathrm{dBm}$, SVR-NLE outperforms by 1 $\mathrm{dB}$ in $\mathrm{Q}$-factor. In addition, it is shown that SVR is significantly less complex than full-field DBP and V-NLE.

Fig. 1 depicts (a) the block diagram of the CO-OFDM receiver equipped with NLE, and (b) the proposed SVR-NLE comprised of $k$ hidden nodes (support vectors) with each node being associated to each subcarrier $k$. The received symbols for each subcarrier $x\{k\}$ are processed by the NLE supported vectors which are scaled by weight 
values (i.e. the Lagrange multipliers) for each subcarrier $\mathrm{w}_{\mathrm{k}, \mathrm{i}}$ after which, the outputs for different $k$ are summed.

Distribution of noisy possible constellation point is learnt during an initial training process. Once these distributions are learnt, the detector can make decision for the new unknown observation symbols. The hyperplane is obtained through approximation of a nonlinear function using a set of Kernels (sigmoid function) of $l$ training dataset $\left\{\left(x^{1}, y^{1}\right),\left(x^{2}, y^{2}\right), \ldots,\left(x^{l}, y^{l}\right)\right\}$. In Fig. 2, an example is depicted for SVR showing how a data-set with noise can be extracted using the Kernel "trick" and thus controlling "overfitting". In Fig. 2(a) the graphical representation depicts a data-set with noise using vectors of +1,-1; and in Fig. 2(b) it is shown how the useful data could be extracted via a powerful Kernel without being corrupted by the noisy data.

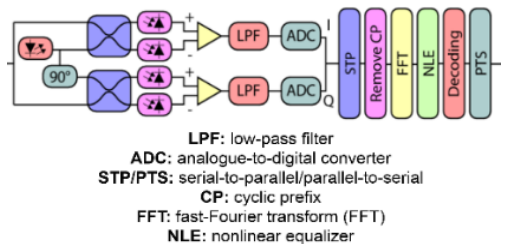

(a)

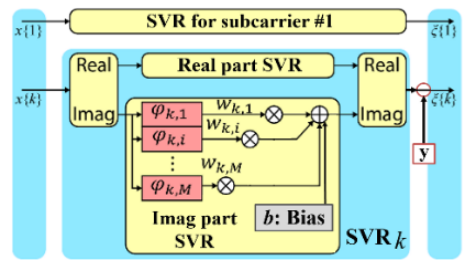

(b)

Fig. 1. (a) Block diagram of the CO-OFDM receiver equipped with NLE. (b) Proposed SVR-NLE.

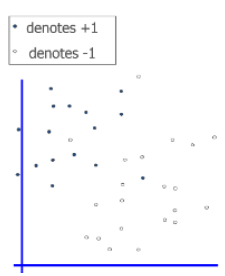

(a)

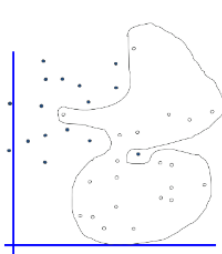

(b)
Fig. 2. SVR example: (a) data-set with noise; (b) example of using powerful Kernel "trick" to distinguish useful data from noisy data.

Afterwards, SVR maps the data to a high-dimension feature space using a nonlinear mapping $\varphi$ (Kernel-based sigmoid function) and then linear regression is formulated by introducing the " $\varepsilon$ insensitive" loss function in the following form:

$$
f(x, w)=\sum_{i=1}^{M} w_{k, i} \varphi_{k, i}(x)+b
$$

where $f(x, w)$ is the target linear model, $\varphi_{k, i}(x)$ denotes a set of nonlinear transformations of input $x$, and $b$ is the bias term. The number of vectors in every hidden node is equal to the number of points of the constellation, i.e. $M$ in (1), which in the case of 16-QAM is 16. Afterwards, (1) can be learnt through training process by minimizing the error:

$$
\psi(w, \xi)=\frac{1}{2}\|w\|^{2}+C \sum\left(\xi_{k}^{-}+\xi_{k}^{+}\right)
$$

where $\xi_{k}^{-}, \xi_{k}^{+}$are slack variables [7] corresponding to the upper and lower bounds on the output function, and $C$ is the penalty parameter which controls the trade-off between the slack variable penalty and the margin [7]. Depending on how much loss is ignored, the solution of (2) can be approximated by the Lagrange-based loss function $L(y, f(x, w))$, and thus, the adopted " $\varepsilon$-insensitive" loss function can be expressed as:

$$
L_{\varepsilon}(y, f(x, \omega))=\left\{\begin{array}{c}
0 \text { if }|y-f(x, w)| \leq \varepsilon \\
|y-f(x, w)|-\varepsilon, \text { otherwise }
\end{array}\right.
$$

The procedure of SVR involves 2 stages:

- Training:

a) Arrange the data to form SVR packet with label (I and Q);

b) Perform (I, Q) data scaling to $[0,1]$;

c) Select the sigmoid Kernel function;

d) Use cross validation to find the best $C$ and the standard single-mode fiber (SSMF)-induced nonlinearity parameter;

e) Use $C$ and nonlinearity parameter to build the SVR for the whole "training set".

- Testing:

a) Approximate functions of the form presented in (1) with " $\varepsilon$-insensitive" loss function. Loss is zero if difference between $f(x, w)$ and measured value is $<\varepsilon$. Vapnik's " $\varepsilon$-insensitivity" loss function defines an $\varepsilon$ tube around $f(x, w)$ [9]. If predicted value is within the tube, the loss (error, cost) is zero while for points outside the loss equals to the magnitude of the difference between the predicted value and the radius $\varepsilon$ of the tube;

b) Compare predicted labels (y-output) to pre-stored transmitted label for bit-error-rate (BER) estimation. In SVR, even if the processing of the initial training sequence might be computational consuming, for a highly stable link, where CD and nonlinear effects do not change over time, the regression coefficients should only be found once. Moreover, there is no oversampling as in V-NLE because SVR-NLE is performed in a subcarrier-by-subcarrier OFDM process.

The block diagram of V-NLE is depicted in Fig. 3, which is similar to $[5,6]$. For V-NLE, $3^{\text {rd }}$ order Volterra Kernels were considered to reduce the complexity, which is identical to that reported in $[5,6]$ to account for single-polarization 16 QAM CO-OFDM. In contrast to SVR-NLE, V-NLE is placed after the analogue-to-digital converters (ADCs) as depicted in Fig. 3 to relax digital signal processing (DSP) complexity by means of reducing the number of inverse fast Fourier transform (IFFT)/FFT blocks. V-NLE inherits some of the features of the hybrid time-and-frequency domain implementation, such as non-frequency aliasing and simple implementation. From Fig. 3, it can be clearly identified that $\mathrm{CD}$, i.e. $\left(H_{C D}\right)^{k}$, and the fiber nonlinearity are combated by the linear and nonlinear compensator tool, respectively. Very high-order Volterra Kernels have not been considered here, thus offering $\sim 50 \%$ reduced computational complexity compared to full-field DBP. 


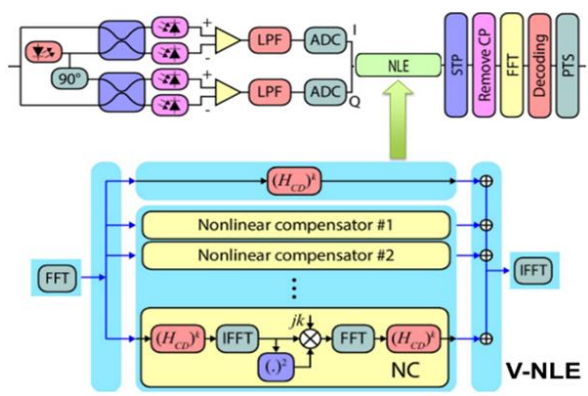

Fig. 3. Block diagram of the CO-OFDM receiver equipped with the benchmark V-NLE. LPF: low-pass filter, ADC: analogue-to-digital converter, STP/PTS: serial-to-parallel/parallel-to-serial, CP: cyclic prefix, (I)FFT: (inverse) fast-Fourier transform, NLE: nonlinear equalizer, NC: nonlinear compensator, $H_{C D}$ : nonlinear system chromatic dispersion.

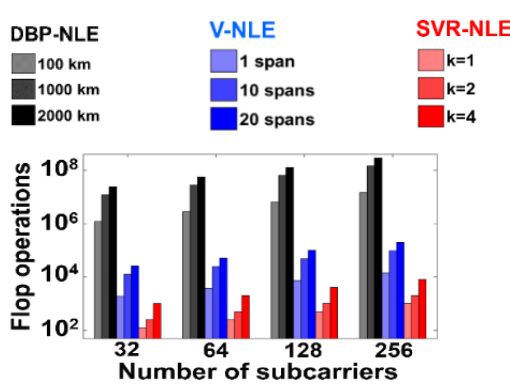

Fig. 4. Computational complexity comparison between SVR-, DBP- and V-NLEs: Blue bars represent the computational complexity of V-NLE for different subcarrier number, $N_{S C}$, and number of spans, $N_{\text {span; }}$; black-grey bars represent the computational complexity of DBP-NLE for different subcarrier number, $N_{S C}$, and transmission lengths, whereas red bars are for SVR-NLE considering various $N_{S c}$, and bits per subcarrier, $N_{b i t s}$ (i.e. $k$ ).

Finally, 200 steps/span (denoted as full-field) were taken for DBP-NLE following procedure similar to [2]. DBP-NLE is also placed after the ADC's in the receiver. The number of floating-point real-valued multiplications required by SVR for decoding each OFDM symbol is:

$$
N_{S V R}=2 \cdot N_{S C}\left(2^{N_{b i t s}}+1\right)
$$

where $N_{S C}$ is the number of subcarriers and $N_{b i t s}$ is the number of bits encoded in each subcarrier. The number of multiplications in full-field DBP is:

$$
N_{D B P}=d_{\text {link }} / d_{\text {step }}\left[8 N_{S C} K \log _{2}\left(N_{S C} K\right)-9 K N_{S C}+16\right]
$$

in which where $d_{\text {link }}$ and $d_{\text {step }}$ are the total link distance and the splitting step, respectively, and $K$ is the oversampling factor. For a system under test with $N_{\text {bits }}=4, N_{S C}=512$, and $d_{\text {link }}=2000 \mathrm{~km}$, and assuming $K=4$ and $d_{\text {step }}=1 \mathrm{~km}$, it is calculated that $N_{S V R}=17408$ whereas $N_{D B P}=145440000$, that is, a difference of $\sim 4$ orders of magnitude. On the other hand, the numbers of multiplications required by V-NLE is: $N_{\text {Volterra }}=\left(N_{\text {span }}+1\right) 8 N_{s c} K \log _{2}\left(N_{s c} K\right)+\left(20 N_{\text {span }}-6\right) N_{s c} K+16\left(N_{\text {span }}+\right.$ $1)$ in which where $N_{\text {span }}$ is the number of spans. The computational complexity of V-NLE depends on $N_{\text {span }}$ but not on $N_{b i t s}$, while as shown from (4) SVR-NLE does not depend on the link-related parameters but on $N_{b i t s}$ since it is sensitive to the number of points in the constellation. Fig. 4 shows a detailed quantitative comparison in terms of $N_{S C}$ for different system parameters. For V-NLE a $K$ of 4 has been set and the $N_{\text {span }}$ has been varied $(1,5$, and 10$)$, for DBP-NLE a $K$ of 4 has also been set and the total link distance ( $d_{\text {link }}$ ) has been varied $(100 \mathrm{~km}, 1000 \mathrm{~km}$, and $2000 \mathrm{~km})$, whereas for SVR-NLE, the $N_{\text {bits }}$ has been swept ( $k=1,2$, and 4$)$. It is shown that, for all considered $N_{S C}$ values, SVR-NLE outperforms both V-NLE and DBP-NLE in terms of computational complexity. Even when comparing the best-case scenario of V-NLE, i.e. only 1 span, to the worst-case scenario of SVR i.e. $4 N_{\text {bits }}$, the latter always outperforms. This difference increases accordingly to the number of spans, which is the case of long-haul networks.

Fig. 5 depicts the experimental setup where an external cavity laser (ECL) of $100 \mathrm{KHz}$ linewidth was modulated using a dual-parallel Mach-Zehnder modulator (DP-MZM) in I-Q configuration. The DP-MZM was fed with OFDM I-Q components, which was generated offline. The transmission path at $1550.2 \mathrm{~nm}$ was a recirculating loop consisting of $20 \times 100 \mathrm{~km}$ spans of Sterlite OH-LITE (E) fiber (attenuation, $\alpha$, of 18.9-19.5 dB/100 km) controlled by acousto-optic modulator (AOM). The loop switch was located in the midstage of the $1^{\text {st }}$ Erbium-doped fiber amplifier (EDFA) and a gain-flattening filter (GFF) was placed in the mid-stage of the $3^{\text {rd }}$ EDFA. The LOP was swept by controlling the output power of the EDFAs. At the receiver, the incoming signal was combined with another $100 \mathrm{KHz}$ linewidth ECL acting as LO. After down-conversion, the baseband signal was sampled using a real-time oscilloscope operating at $80 \mathrm{GS} / \mathrm{s}$ and processed offline in MATLAB $^{\circledR} .400$ OFDM symbols were generated using a 512-point IFFT, 210 subcarriers were modulated using 16-QAM, while the rest were set to zero. To eliminate the PMD-induced inter-symbol-interference, a cyclic prefix (CP) of $2 \%$ was included. The SVR training overhead was set at $10 \%$ similarly to [7] resulting in a training length of 40 symbols. The net bit-rate was $\sim 40-\mathrm{Gb} / \mathrm{s}$. The offline OFDM demodulator included both timing synchronization and frequency offset compensation, as well as I-Q imbalance and CD compensation using an overlapped frequency domain equalizer employing the overlap-and-save

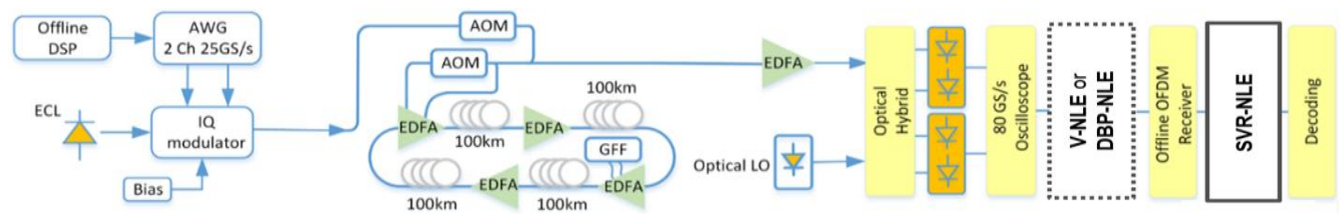

Fig. 5. Experimental setup of 40-Gb/s CO-OFDM equipped with NLE. ECL: external cavity laser, DSP: digital signal processing, AWG: arbitrary waveform generator, AOM: acousto-optic modulator, EDFA: Erbium-doped fiber amplifier, GFF: gain flatten filter, LO: local oscillator. 
method. The raw bit-rate for all techniques including linear equalization was $\sim 45.6-\mathrm{Gb} / \mathrm{s}$. NLE was assessed by Q-factor measurements averaging over 10 recorded traces $\left(\sim 10^{6}\right.$ bits), which was estimated from the BER obtained by error counting after hard-decision decoding. The Q-factor is related to BER by: $\mathrm{Q}=20 \log _{10}\left[\sqrt{2} \operatorname{erfc} c^{-1}(2 B E R)\right]$. For 16QAM, a BER of $10^{-3}$ (FEC-limit) results in a $\mathrm{Q}$-factor of $\sim 9.8$ dB. In Fig. 6, the Q-factor against the LOP is plotted for 40 $\mathrm{Gb} / \mathrm{s}$ CO-OFDM at $2000 \mathrm{~km}$ for SVR-NLE, DBP-NLE, V-NLE and without (w/o) employing NLE. It is shown that SVR-NLE can extend the optimum LOP by $4 \mathrm{~dB}$ compared to both linear equalization and V-NLE by means of reduction of fiber nonlinearity. In comparison to full-field DBP-NLE at a LOP of $6 \mathrm{dBm}$, SVR-NLE outperforms by $\sim 1 \mathrm{~dB}$ in Q-factor. This occurs due to the inability of both V-NLE and DBP-NLE to combat the stochastic-induced inter-subcarrier intermixing based effects of four-wave mixing and cross-phase modulation.

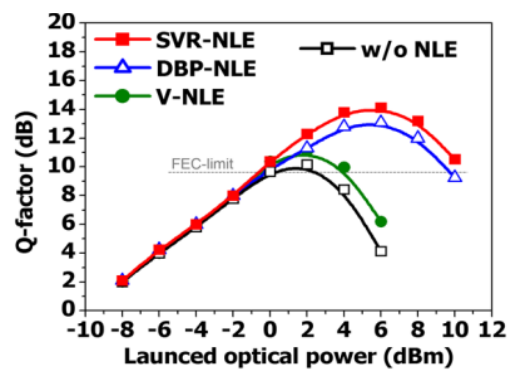

Fig. 6. Q-factor vs. launched optical power (LOP) for SVR-NLE, DBP-NLE, V-NLE and without (w/o) NLE, for 40-Gb/s 16-QAM CO-OFDM at 2000 $\mathrm{km}$ of transmission.

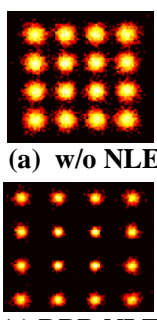

(c) DBP-NLE

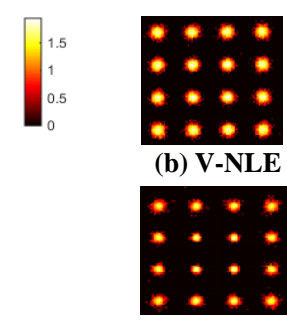

(d) SVR-NLE
Fig. 7. Received 16-QAM constellation diagram of 40-Gb/s CO-OFDM for a LOP of $4 \mathrm{dBm}$ (a) w/o NLE [Q-factor $=8.2 \mathrm{~dB}$ ], (b) with V-NLE [Q-factor $=10.4 \mathrm{~dB}$ ], (c) with DBP-NLE [Q-factor $=12.5 \mathrm{~dB}$ ] and (d) with SVR-NLE $[\mathrm{Q}$-factor $=14 \mathrm{~dB}]$. Inset: Colour bar.

In Fig. 7, a comparison is depicted between the received 16-QAM constellations of 40-Gb/s CO-OFDM at a LOP of $4 \mathrm{dBm}$ for all three aforementioned DSP-NLEs and w/o NLE. From these constellations it is evident that SVR-NLE condenses the constellation points more effectively in contrast to the two benchmark deterministic NLEs (i.e. V-NLE and DBP-NLE) by decreasing the symbol dispersion due to its stochastic-induced nonlinearity mitigation capability.

In conclusion, a novel SVR based NLE was experimentally compared to V-NLE and full-field DBP-NLE in 40-Gb/s 16-QAM COOFDM at $2000 \mathrm{~km}$. SVR-NLE extended the optimum LOP by $4 \mathrm{~dB}$ compared to V-NLE and outperformed by $\sim 1 \mathrm{~dB}$ in Q-factor to fullfield DBP-NLE at a LOP of $6 \mathrm{dBm}$. Moreover, SVR-NLE is significantly less complex than both full-field DBP-NLE and V-NLE.
Funding. Centre of Excellence (CE110001018); Laureate Fellowship (FL120100029); EPSRC (EP/J017582/1, EP/L000091/1); FAPESP (2015/04113-0).

\section{References}

1. I. D. Phillips, M. Tan, M. F. C. Stephens, M. E. McCarthy, E. Giacoumidis, S. Sygletos, P. Rosa, S. Fabbri, S. T. Le, T. Kanesan, S. K. Turitsyn, N. J. Doran, P. Harper, A.D. Ellis, in OFC (OSA, 2014), p. M3C.1.

2. G. Gao, J. Zhang, and W. Gu, IEEE Photon. Technol. Lett. 25, 717 (2013).

3. E. Temprana, E. Myslivets, B.P.-P. Kuo, L. Liu, V. Ataie, N. Alic, and S. Radic, Science 348, 1409 (2015).

4. X. Liu, A. R. Chraplyyy, P. J. Winzer, R. W. Tkach, and S. Chandrasekhar, Nature Photon. 7, 560 (2013).

5. E. Giacoumidis, I. Aldaya, M. A. Jarajreh, A. Tsokanos, S. T. Le, F. Farjady, A. D. Ellis, and N. J. Doran, IEEE Photon. Technol. Lett. 26, 1383 (2014).

6. M. A. Jarajreh, E. Giacoumidis, I. Aldaya, S. T. Le, A. Tsokanos, Z. Ghassemlooy, and N. J. Doran, IEEE Photon. Technol. Lett. 27, 387 (2015).

7. M. Li, S. Yu, J. Yang, Z. Chen, Y. Han, W. Gu, IEEE Photon. J. 5, (2013).

8. S. T. Le, M. E. McCarthy, N. Mac-Suibhne, M. Al-Khateeb, E. Giacoumidis, N. J. Doran, A. D. Ellis, S. K. Turitsyn, IEEE J. Lightw. Technol. 33, 2206 (2015).

9. V. Vapnik, Secaucus, NJ, USA: Springer-Verlag New York, Inc., 1982.

\section{Full References Description}

1. D. Phillips, M. Tan, M. F. C. Stephens, M. E. McCarthy, E. Giacoumidis, S. Sygletos, P. Rosa, S. Fabbri, S. T. Le, T. Kanesan, S. K. Turitsyn, N. J. Doran, P. Harper, A.D. Ellis, "Exceeding the nonlinear-Shannon limit using Raman laser based amplification and optical phase conjugation", OSA Optical Fiber Communication Conference and Exposition and the National Fiber Optic Engineers Conference (OFC/NFOEC), San Francisco, California, USA, Paper M3C.1

2. G. Gao, J. Zhang, and W. Gu, "Analytical evaluation of practical DBP-based intra-channel nonlinearity compensators," IEEE Photon. Technol. Lett. 25, 717 (2013).

3. E. Temprana, E. Myslivets, B.P.-P. Kuo, L. Liu, V. Ataie, N. Alic, and S. Radic, "Overcoming Kerr-induced capacity limit in optical fiber transmission", Science 348, 1409 (2015).

4. X. Liu, A. R. Chraplyy, P. J. Winzer, R. W. Tkach and S. Chandrasekhar, "Phase-conjugated twin waves for communication beyond the Kerr nonlinearity limit", Nature Photon. 7, 560 (2013).

5. E. Giacoumidis, I. Aldaya, M. A. Jarajreh, A. Tsokanos, S. T. Le, F. Farjady, A. D. Ellis, and N. J. Doran, "Volterra-based reconfigurable nonlinear equalizer for dual-polarization multiband coherent OFDM", IEEE Photon. Technol. Lett. 26, 1383 (2014).

6. M. A. Jarajreh, E. Giacoumidis, I. Aldaya, S. T. Le, A. Tsokanos, Z. Ghassemlooy, and N. J. Doran, "Artificial neural network nonlinear equalizer for coherent optical OFDM", IEEE Photon. Technol. Lett. 27, 387 (2015).

7. M. Li, S. Yu, J. Yang, Z. Chen, Y. Han, W. Gu, “Nonparameter Nonlinear Phase Noise Mitigation by Using M-ary Support Vector Machine for Coherent Optical Systems", IEEE Photon. J. 5 (2013).

8. S. T. Le, M. E. McCarthy, N. Mac-Suibhne, M. Al-Khateeb, E. Giacoumidis, N. J. Doran, A. D. Ellis, S. K. Turitsyn, “Demonstration of Phase-conjugated Subcarrier Coding for Fiber Nonlinearity Compensation in CO-OFDM Transmission", IEEE J. Lightw. Technol. 33, 2206 (2015).

9. V. Vapnik, Estimation of Dependences Based on Empirical Data: Springer Series in Statistics (Springer Series in Statistics), Secaucus, NJ, USA: SpringerVerlag New York, Inc., 1982. 